\title{
Combined Riccati-Genetic Algorithms Proposed for Non-Convex Optimization Problem Resolution - A Robust Control Model for PMSM
}

\author{
Khira DCHICH, Abderrahmen ZAAFOURI, Abdelkader CHAARI \\ University of Tunis, Unit C3S, \\ Higher School of Sciences and Techniques of Tunis (ESSTT), \\ 5 Av. Taha Hussein, BP 56, 1008 Tunis, TUNISIA \\ Khira.Dchich@fsb.rnu.tn, abderrahmen.zaafouri@isetr.rnu.tn, assil.chaari@esstt.rnu.tn
}

\begin{abstract}
In this paper, is proposed a state feedback optimal control algorithm for uncertain linear systems, with norm bounded uncertainties. It is based on the use of Algebraic Riccati Equation - Genetic Algorithm (ARE-GA) developed for non-convex optimization problem resolution. The case of an uncertain Permanent Magnet Synchronous Motor (PMSM) based on the use of an Extended Kalman Filter (EKF) to estimate both position and speed, without any mechanical sensor is considered to illustrate the efficiency of the proposed technique.
\end{abstract}

Keywords: Quadratic Stabilization, Uncertain System, Riccati Equation, Convex, Non-convex, Genetic Algorithm, Permanent Magnet Synchronous Motor, Extended Kalman Filter.

\section{Introduction}

In 1993, Rockafellar did affirm that "the great watershed in optimization is not between linearity and nonlinearity, but convexity and non-convexity" [1]. The main objective of this work is to apply this sentence in the context of the convex optimization and non-convex optimization field [2].

The optimization provides a rich algorithmic framework for all areas of applied sciences. There are two branches of deterministic optimization: convex programming and non-convex programming. A convex optimization problem is defined by the minimization of a convex function (objective) within convex constraints. When the double convexity, in the objective and the constraints, is not satisfied the problem falls into non-convex optimization field.

In this paper is presented a new approach to solve the problem of quadratic stabilizability of uncertain linear systems. The proposed idea is to split the problem in two parts: a convex part, involving a large number of decision variables that requires solving an Algebraic Riccati Equation (ARE) [6],[7], and a non-convex part, involving a small number of decision variables, which solution is estimated using a Genetic Algorithm (GA) [8]-[10]. Genetic algorithms [8] are an optimization technique developed in 1975 by J. Holland that have been inspired by Charles Darwin's theory of biological population's evolution. Genetic algorithms are based on the principle of the survival of the fittest, which means that the best suited structures and the closest to the desired result, using genetic operators such as selection, crossover and mutation. The fitness of a particular individual is measured using a fitness function, which evaluates how close the individual is to the objective [9]-[12].

When using a quadratic Lyapunov function, it is possible to synthesize a control law which takes into account the variations of uncertainty that guarantee the quadratic stability of a closed loop system [13]-[15].

The approaches are applied to determine adequate control laws for an uncertain Permanent Magnet Synchronous Motor (PMSM). Controlled PMSM performances depend on the applied control law, the parameters uncertainties and the existence of position and speed sensors.

The objective is to improve the performance of mechanical sensorless vector control of Permanent Magnet Synchronous Machine (PMSM) [3]. This control law requires accurate knowledge of rotor position that provides the autopilot of the machine, which can be obtained directly by a position sensor or indirectly by a speed sensor.

The inherent advantages related to the use of mechanical sensors, placed on the shaft of the machine, are multiple [4], [5].

Considering all these limitations that presents the machine with a mechanical sensor, numerous studies have been made to remove the mechanical sensor while maintaining the proper functioning of the machine [3], [5]. These studies have shown 
different methods of sensorless vector control. To estimate the position and the speed of the machine, a mathematical model should be represented based on the electrical parameters as currents and voltages. One of the method for estimation is the Kalman filter adapted for a robust control in order to get maximum variables for observation. The Kalman filter algorithm deploys the parameters of the machine in order to get a minimization error on the state estimation. Moreover, such a method is well known for the easy implementation especially with the permanent magnet synchronous machine. In this paper, the measured currents and voltages are transformed in the Clark referential and the speed and the position are estimated by the use of the Kalman filter algorithm.

When the parameters of the PMSM are uncertain, [3], [29] and the state variables estimated by EKF [4], a robust feedback control law [29] is well adapted and good performances can be obtained by using a AREGA approach.

The organisation of the paper is as follows. The quadratic stabilizability problem of an uncertain linear system is given in the second section. Then, in the third section, the application of the convex optimization approach using Riccati solvers (ARE) is presented.

The ARE solvers and the genetic algorithms (GA), introduced in section 4, are used to solve the problem split into a convex and a non convex subproblem and to optimize the $\mathrm{P}$ and $\varepsilon$ parameters of the Riccati matrix Equation.

The studied servo-motor model diagram and its Park model are given in section 5, then, the extended Kalman filter algorithm is introduced to estimate the position and the speed from the stator phase currents and voltage in Section 6 then 7. The approaches are applied, with success, to determine adequate control laws for the parameters uncertainties of PMSM, in Section 8 .

\section{Quadratic Stabilization of Uncertain Systems. Problem Statement}

Consider the following linear system, described in state space by

$$
\left\{\begin{array}{l}
x(t)=A x(t)+B u(t) \\
y(t)=C x(t)
\end{array}\right.
$$

$x(t) \in R^{n}$ is the state vector, $u(t) \in R^{n}$ is the control vector, $A \in R^{m \times n}$ and $B \in R^{m \times n}$ constant matrices, such that $(A, B)$ is a controllable pair and $B$ has full column rank.

The state feedback control law has the following general form

$u(t)=K x(t)$

the closed-loop system can be described by

$\dot{x}(t)=(A+B K) x(t)$

The design of this control law, minimizing the following cost function $J$ [16]

$J=\int_{0}^{\infty}\left(x^{T} Q x+u^{T} R u\right) d t$

where $Q \geq 0$ and $R>0$ are, respectively, the state and the input weighting matrices of the criterion $J$, needs the resolution of an Algebraic Ricatti Equation (ARE) in the form

$A^{T} P+P A-P B R^{-1} B^{T} P+Q=0$

It comes the solution $P$ of equation (5) and the control gain $K$ such that

$K=R^{-1} B^{t} P$

For computing the gain $\mathrm{K}$, the matrices $\mathrm{Q}$ and $\mathrm{R}$ should be introduced. Once we formulate the closed loop characteristic matrix, the eigenvalues and eigenvectors will be used as the new performances of the system.

In the case where the uncertainty is in norm bounded type, the system (1) can be described by [5], [17]

$\dot{x}(t)=(A+\Delta A) x(t)+B u(t)$

where $\triangle A$ represents the uncertainty of the studied system such that

$\triangle A=D F E$

$D$ and $E$ are constant matrices of appropriate sizes and $F$ a matrix satisfying the following matrix inequality:

$F^{T} F \leq 1$

The stability of the resulting closed-loop uncertain system (7) is then established by the use of a quadratic Lyapunov function.

\subsection{Definition}

The uncertain linear system (7) is said to be quadratically stabilizable, if there exists a linear feedback control law (2), a positive semidefinite symmetric matrix $P \in R^{n \times n}$ and a 
constant parameter $\alpha>0$ such that the following condition holds: Given any admissible uncertainly $F$, the Lyapunov function $V(x)=x^{T} P x$ is such that [17]

$$
\dot{V}=x^{T}\left[A^{T} P+P A\right] x+2 x^{T} P D F E x \leq-\alpha\|x\|^{2}
$$

The synthesis of such control law algorithm, needing the resolution of an algebraic Riccati equation, is proposed in [17] where is introduced a necessary and sufficient condition for quadratic stabilizability by linear state feedback.

\subsection{Theorem}

The uncertain linear system (7) is said to be quadratically stabilizable, if there exists a linear feedback control law (2), a constant $\varepsilon$ such that, for any positive-definite symmetric matrix $R$, the Riccati equation

$$
\begin{aligned}
& A^{T} P+P A-P B R^{-1} B^{T} P+ \\
& +\varepsilon P D D^{T} P+\varepsilon^{-1} E^{T} E+Q=0
\end{aligned}
$$

or the inequalities

$$
\begin{aligned}
& A^{T} P+P A-P B R^{-1} B^{T} P+ \\
& +\varepsilon P D D^{T} P+\varepsilon^{-1} E^{T} E<0
\end{aligned}
$$

have a positive semi-definite symmetric matrix solution $P \in R^{n \times n}$, expressed by (6).

For fixed parameter $\varepsilon$, the resolution of equation (11) corresponds to a convex problem which becomes non-convex, for free $\varepsilon$.

\subsection{Problem formulation}

Find the parameters $P$ and $\varepsilon$, solutions of equation (11) minimizing the criterion (4). A, $B, Q$ and $R$ are respectively real matrices of dimensions $n \times n, n \times m, n \times n$ and $m \times m, Q$ a symmetric positive semi-definite matrix and $R$ a symmetric positive definite matrix.

\section{Convex Optimization Problem Resolution Algorithm}

Many problems of uncertain systems can be solved through convex optimization tools. In this case, the computation time to find a solution is reasonable; the result corresponds to a global minimum of the criterion [18], [32].

Let a function $f: E \subset \mathfrak{R}^{n} \rightarrow \mathfrak{R}$ such that $E$ is a convex set, Figure 1.

$$
f\left(\lambda x_{1}+(1-\lambda) x_{2}\right) \leq \lambda f\left(x_{1}\right)+(1-\lambda) f\left(x_{2}\right)
$$

$\forall \lambda \in[0,1] \subset \Re, \quad \forall\left(x_{1}, x_{2}\right) \in E^{2} \quad$ and $\quad \lambda \quad$ a constant parameter.

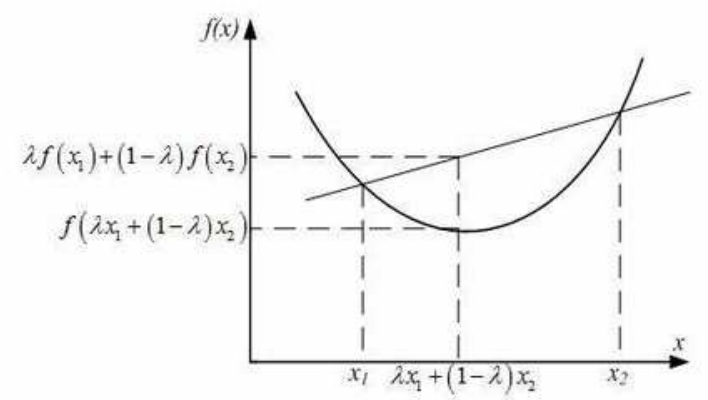

Figure 1. Convex function

\subsection{Proposed algorithm}

The stabilization approach by the control law of the process (7), defined by (2), required for the resolution of the Riccati equation (11), follows the following steps

1. choose the weighting matrices $Q$ and $R$ such that $R=Q=I$ and take,

2. solve the equation (11); if the solution, the system is quadratically stabilizable; then, calculate $K$. Otherwise, go to 3 .,

3. replace by ; if , stop; the system is not quadratically stabilizable. Otherwise, return to 2 ..

This algorithm is used, in section 8 , for the parameters uncertainties of PMSM.

\section{Non-Convex Optimization Problem Resolution Algorithm}

Consider the problem of finding a positive semi-definite solutions to the Riccati equation (11) which appears often in a wide range of control applications.

In the case of an $n^{\text {th }}$-order system, the parameters to determine are $\varepsilon>0$ and $P>0$, $P=\left\{p_{i j}\right\}$, such that $p_{i j}=p_{j i} \forall i, j$.

The optimization problem to solve becomes the following one.

$$
\begin{gathered}
\text { minimize } \varepsilon \\
\text { subject to: } \\
A^{T} P+P A-P B R^{-1} B^{T} P+\varepsilon P D D^{T} P+\varepsilon^{-1} E^{T} E<0 \\
P>0
\end{gathered}
$$

For any fixed value $\varepsilon=\varepsilon_{0}$, the problem is convex and can be solved via Riccati solvers and the solution $P$, which depends on 
$N=1 / 2(n+1) n$ decision parameters $p_{i j}$, $\forall i, j$, determined.

Then, for non fixed $\varepsilon$, the problem is nonconvex and cannot be solved using standard solvers. This observation motivates the idea to split the original problem into a small nonconvex part solved by Genetic Algorithm (GA) and a large convex part solved with a Riccati solver, as shown in Figure 1. Then, to do this, we propose [7] to

- let a fast and efficient Riccati solver takes care of the large convex part of the problem: for a given find the unique solution $\mathrm{P}$ (if it exists), and

- let GA, which may be unreliable for a large number of decision parameters, deal with the smaller non-convex part and search over (which usually depends on the controller and other parameters)
Thus, GA is used to construct the constant $\varepsilon$ and a Riccati solver applied to calculate $P$ (if it exists). The full chromosomes are constructed by adjoining the decision parameters in and $P$.If a standard GA is used alone to solve the original problem, the GA chromosomes must code both $\varepsilon$ and $P$, and if $P$ is large, the chromosome, consequently, will be too long for an efficient and reliable solution.

Reducing the dimension of the solution space for the GA which doesn't only accelerate the evolution process, but also increases the chances of converging to the global solution of the problem. The overall algorithm is given in Figure 2. Once the full chromosomes are constructed and the fitness evaluated.

It is applied, in the next section, to the uncertain PMSM study and its efficiency compared to ARE approach.

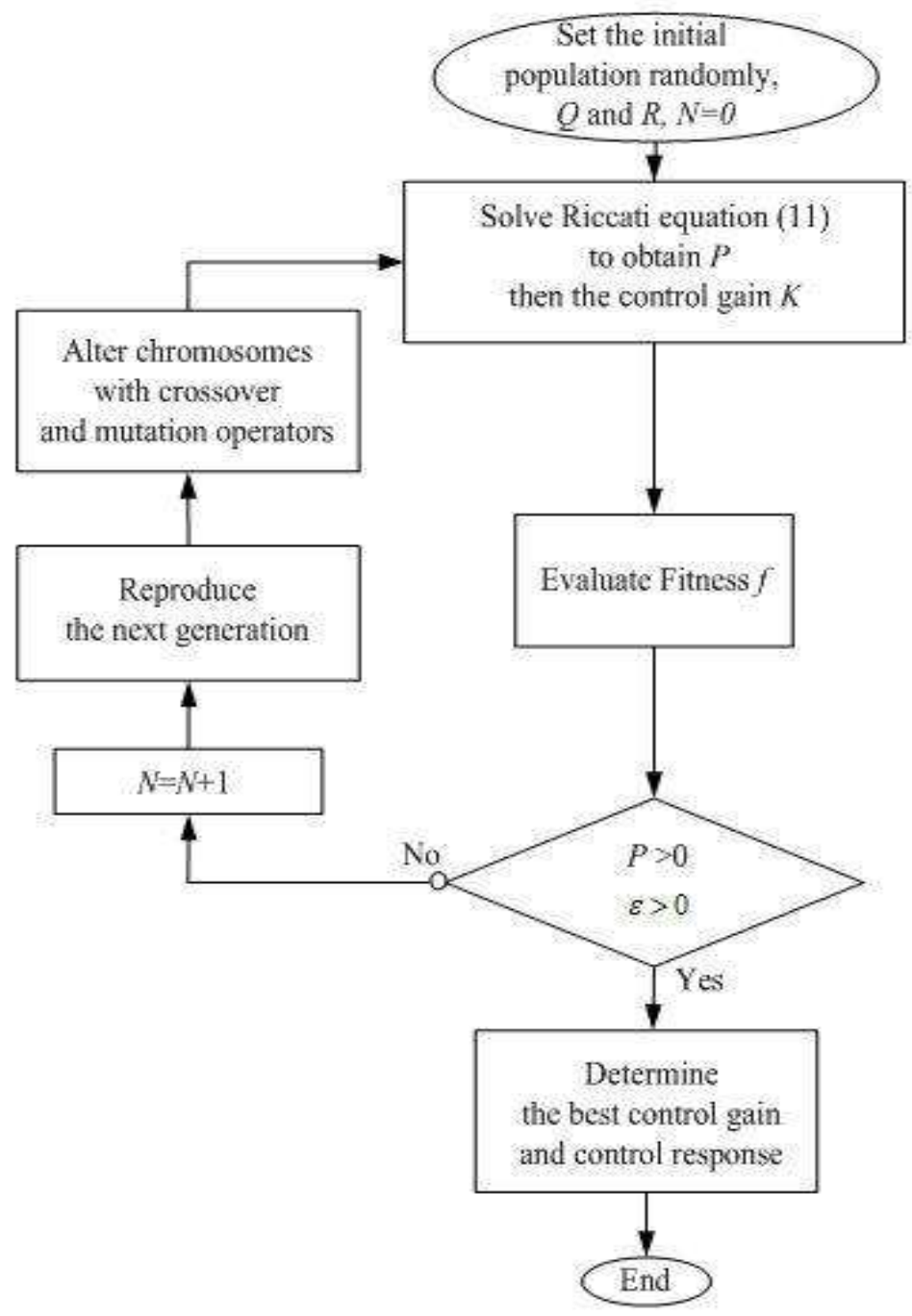

Figure 2. The structure of the proposed ARE-GA algorithm 


\section{Studied Synchronous Machine with Permanent Magnets Case}

Let us consider the salient-pole PMSM drive system, given in Figure 3.

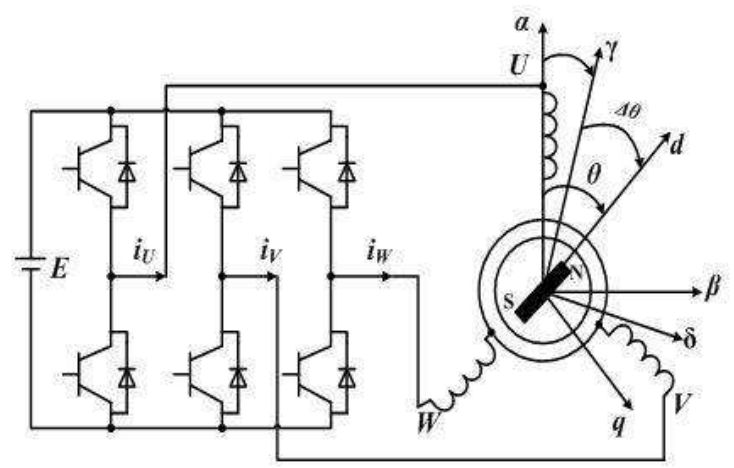

Figure 3. Servo-motor analytical model diagram

It shows:

- the orthogonal two-phases ( $\alpha$ and $\beta$ ) frame fixed to the stator windings,

- the (d and q) frame showing the synchronously rotating reference frame,

- the d-axis coincides with the $\mathrm{N}$ poles of the rotor, $\mathrm{q}$ representing the rotor angular position.

The dynamic model of the PMSM is developed according to some simplifying hypotheses [3], [4]. Thus, saturation and iron losses are not considered. The back emf is assumed to have a sine form, while eddy currents are neglected. Permanent magnets are buried in the rotor steel; so, the machine is characterized by a cylindrical asymmetry causing the direct axes inductance $L_{d}$ to be lower than the quadrature one $L_{q}$.

The dynamic $d-q$ model for a salient-pole PMSM, in the rotor reference frame, is given as follows

$\left[\begin{array}{l}V_{d} \\ V_{q}\end{array}\right]=\left[\begin{array}{cc}R+L_{d} s & -L_{q} \omega \\ L_{d} \omega & R+L_{q} s\end{array}\right]\left[\begin{array}{l}l_{d} \\ l_{q}\end{array}\right]+\left[\begin{array}{c}0 \\ K_{t} \omega\end{array}\right]$

$s$ is the Laplace operator.

The following relation limits both marks $\gamma-\delta$ and $d-q$

$\left[\begin{array}{l}x_{\lambda} \\ x_{\delta}\end{array}\right]\left[\begin{array}{cc}\cos \Delta \theta & -\sin \Delta \theta \\ \sin \Delta \theta & \cos \Delta \theta\end{array}\right]\left[\begin{array}{l}x_{d} \\ x_{q}\end{array}\right]$

where $x$ represents either the current, the voltage or the flux.
The stator flux equations of the synchronous machine with magnets in the $d-q$ axes, linked to the rotor, are written such as

$$
\begin{aligned}
& \Phi_{d}=L_{d} i_{d}+K_{t} \\
& \Phi_{d}=L_{q} i_{q}
\end{aligned}
$$

with

$$
\begin{aligned}
& K_{t}=\sqrt{\frac{3}{2}} \Phi_{f}, \quad L_{d}=I_{s}+\frac{3}{2}\left(L_{0}-L_{2}\right), \quad \omega=\frac{d \theta}{d t}, \\
& L_{d}=I_{s}+\frac{3}{2}\left(L_{0}+L_{2}\right) .
\end{aligned}
$$

and

$L_{d}$ : synchronous cyclic inductance of direct axis,

$L_{q}$ : cyclic inductance of the transversal axis,

$\widehat{\Phi}_{f}$ : inductor flux created by the magnets,

$l_{s}:$ leak inductance,

$L_{0}$ : constant term of the proper inductance of the induced winding,

$L_{2}$ : amplitude of the harmonious term order 2 of the proper inductance of the induced.

The electromagnetic torque is defined by

$C_{e}=n_{p}\left(\Phi_{d} i_{q}-\Phi_{q} i_{d}\right)$

then, by substituting (16) in (17), by

$C_{e}=n_{p}\left[\left(L_{d}-L_{q}\right) i_{d} i_{q}+K_{t} i_{q}\right]$

$n_{p}$ is the pole pair number of the studied machine.

The mechanical equation is given by the following differential equation

$J \frac{d \Omega}{d t}+f \Omega=C_{e}-C_{r}$

with

$f$ : moment of inertia of the rotating part,

$J$ : coefficient of viscous friction,

$C_{r}$ : load torque.

For $i_{d}$ equal to zero, the electromechanical torque $C_{e}$ becomes proportional to the stator current component $i_{q}$, such as

$C_{e}=n_{p} K_{t} i_{q}$

This is relation is similar for a machine with a continuous current. Then, PMSM can be described by the following Park model. 


$$
\begin{aligned}
& \left|\begin{array}{c}
\frac{d i_{d}}{d t} \\
\frac{d i_{q}}{d t} \\
\frac{d \omega}{d t} \\
\frac{d \theta}{d t}
\end{array}\right|=\left[\begin{array}{cccc}
\frac{-1}{\tau_{d}} & \frac{-L_{d}}{L_{q}} \omega & 0 & 0 \\
\frac{-L_{d}}{L_{q}} \omega & \frac{-1}{\tau_{d}} & \frac{-K_{e}}{L_{q}} & 0 \\
n_{p}^{2} \frac{L_{d}-L_{q}}{J} & n_{p}^{2} \frac{K_{t}}{J} & \frac{-f}{J} & 0 \\
0 & 0 & 1 & 0
\end{array}\right]\left[\begin{array}{c}
i_{d} \\
i_{q} \\
\omega \\
\theta
\end{array}\right]+ \\
& +\left[\begin{array}{ccc}
\frac{-1}{L} & 0 & 0 \\
0 & \frac{1}{L_{d}} & 0 \\
0 & 0 & -\frac{n_{p}}{J} \\
0 & 0 & 0
\end{array}\right]\left[\begin{array}{c}
v_{d} \\
v_{q} \\
C_{r}
\end{array}\right]
\end{aligned}
$$

where $\tau_{d}=\frac{L_{d}}{R}$ and $\tau_{d}=\frac{L_{q}}{R}$ are, respectively, the time constants of direct axis and in squaring, and $v_{q}$ the control inputs, $C_{r}$ a disturbance input and $K_{t}$ a emf constant.

\section{Extended Kalman Filter Algorithm}

The Kalman filter is a mathematical tool able to estimate the non measurable state variables from measurable physics parameters [3], [4].

This filter supposes that the noises, which affect the model, are centered and white, and the state noises have to be uncorrelative with the measure noises.

Consider the following stochastic non linear model

$$
\left\{\begin{array}{c}
x(k+1)=f(x(k), U(k))+w(k) \\
z(k)=h(x(k))+v(k)
\end{array}\right.
$$

with $\mathrm{x}(\mathrm{k}) ; \mathrm{U}(\mathrm{k}) ; \mathrm{z}(\mathrm{k})$ : state / control / output vectors, $f(.) ; h($.$) : nonlinear vectors, w(k)$ : state noise vector, $\mathrm{v}(\mathrm{k})$ : measure noise vector.

The procedure is split in a prediction step and a correction step of the considered $\mathrm{x}$ estimation.

\subsection{Prediction step}

Estimation under the prediction form

$$
\hat{x}((k+1) / k)=F(k) \hat{x}\left(\frac{k}{k}\right)+G(k) U(k)
$$

with

$$
F(k)=\left.\frac{\partial}{\partial x}\{f(x(k)), U(k), k\}\right|_{\hat{x}(k), U(k)}
$$

$$
G(k)=\left.\frac{\partial}{\partial U}\{f(x(k)), U(k), k\}\right|_{\hat{x}(k), U(k)}
$$

and F: partial derivative system matrix, G: weighting matrix of noise.

At the instant $\mathrm{k}+1$ this step allows to build a first estimation of the state vector.

Covariant matrix of the prediction error determination

$$
P((k+1) / k)=F(k) P\left(\frac{k}{k}\right) F^{T}(k)+Q
$$

with $P$ : state covariance matrix, $Q$ : system noise covariance matrix.

\subsection{Correction step}

The phase of prediction allows having a gap between the predicted output and the measured output $z(k+1)$. We have to minimize that gap and correct it by the use of the filter gain to improve the state.

Kalman filter gain is characterized by

$$
\begin{aligned}
& K(k+1)=P((k+1) / k) H^{T}(k+1) \times \\
& \times\left(H(k) P((k+1) / k) H^{T}(k)+R\right)^{-1}
\end{aligned}
$$

with

$$
H(k)=\frac{\partial h(x(k))}{\partial x(k)} \mid x(k)-\dot{x}(k)
$$

$\mathrm{R}$ : measurement noise covariance matrix. The covariant matrix defined by

$$
\begin{aligned}
P((k+1) /(k+1)) & =P((k+1) / k)- \\
-K(k+1) & \times H(k+1) P((k+1) / k)
\end{aligned}
$$

and the state vector at the instant $k+1$ by

$$
\begin{array}{r}
\hat{x}((k+1) /(k+1))=\hat{x}((k+1) / k)+K(k+1) \times \\
\times(Z(k+1)-H(k+1) \hat{x}((k+1) / k))
\end{array}
$$

For the simulation, The extended Kalman filter algorithm is inserted in the global simulation system diagram implanted as an "S-function".

\section{Sensorless Control with EKF}

The proposed PMSM controller is based on the use of the stator phase currents and voltages to estimate the position and the speed by EKF [4].

Let the studied machine described in the Clark referential $(\alpha, \beta)$ by the following equations 


$$
\begin{aligned}
{\left[\begin{array}{l}
v_{\alpha} \\
v_{\beta}
\end{array}\right] } & =\left[\begin{array}{ll}
t_{11} & t_{12} \\
t_{21} & t_{22}
\end{array}\right]\left[\begin{array}{l}
i_{\alpha} \\
i_{\beta}
\end{array}\right]+\left[\begin{array}{ll}
f_{11} & f_{12} \\
f_{21} & f_{22}
\end{array}\right]\left[\begin{array}{l}
d i_{\alpha} / d t_{t} \\
d i_{\beta} / d t_{t}
\end{array}\right]+ \\
& +\left[\begin{array}{r}
-\omega K_{t} \sin \theta \\
\omega K_{t} \sin \theta
\end{array}\right]
\end{aligned}
$$

with

$$
\begin{aligned}
& t_{11}=R_{1}-\omega L_{\varepsilon} \sin 2 \theta, \\
& t_{12}=\omega L_{\varepsilon} \cos 2 \theta, \\
& t_{21}=\omega L_{\varepsilon} \cos 2 \theta, \\
& t_{22}=R_{1}+\omega L_{\varepsilon} \sin 2 \theta, \\
& f_{11}=\frac{L_{\varepsilon}}{2}+\frac{L_{\Delta}}{2} \cos 2 \theta, \\
& f_{12}=\frac{L_{\Delta}}{2} \sin 2 \theta, \\
& f_{21}=\frac{L_{\Delta}}{2} \sin 2 \theta, \\
& f_{22}=\frac{L_{\varepsilon}}{2}-\frac{L_{\Delta}}{2} \cos 2 \theta, \\
& L_{\varepsilon}=L_{d}+L_{q}, \\
& L_{\Delta}=L_{d}-L_{q} .
\end{aligned}
$$

The electromagnetic torque in the stationary referential $(\alpha, \beta)$ can be expressed by the relation

$$
C_{e}=n_{p}\left\{\begin{array}{l}
K_{t}\left[i_{p} \cos \theta-i_{\alpha} \sin \theta\right] \\
\frac{+1}{2} L_{D}\left[\left(i_{\beta}^{2}-i_{\beta}^{2}\right) \sin 2 \theta+2 i_{\alpha} i_{\alpha} \cos 2 \theta\right]
\end{array}\right.
$$

For the referential $(\alpha, \beta)$ linked to stator, the description of the PMSM (18) becomes

$$
\frac{d y}{d t}=A_{y}+B_{v}
$$

with,

$$
y=\left[i_{\alpha}, i_{\beta}, \omega, \theta\right]^{T}, \quad v=\left[v_{\alpha}, v_{\beta}, C_{r}\right]^{T}, A \text { an } 4 \times 4
$$
matrix, $A=\left\{a_{i j}\right\}$ and $B$ an $4 \times 3$ matrix, $B=\left\{b_{i j}\right\}$, such that

$$
\begin{aligned}
& a_{11}=\frac{-R}{2 L_{\pi}}\left(L_{\Sigma}-L_{\Delta} \cos 2 \theta\right)+\frac{\omega L_{\Sigma}}{2 L_{o}} L_{\Delta} \sin 2 \theta, \\
& a_{12}=\frac{-\omega L_{\Delta}}{2 L_{o}}\left(L_{\Delta}-L_{\Sigma} \cos 2 \theta\right)+\frac{R}{2 L_{o}} L_{\Delta} \sin 2 \theta, \\
& a_{21}=\frac{-\omega L_{\Delta}}{2 L_{o}}\left(L_{\Delta}+L_{\Sigma} \cos 2 \theta\right)+\frac{R}{2 L_{o}} L_{\Delta} \sin 2 \theta, \\
& a_{22}=\frac{-R}{2 L_{o}}\left(L_{\Sigma}-L_{\Delta} \cos 2 \theta\right)-\frac{\omega L_{\Sigma}}{2 L_{o}} L_{\Delta} \sin 2 \theta,
\end{aligned}
$$

$$
\begin{aligned}
& a_{32}=\frac{-n_{0}^{2}}{J}\left[K_{t} \sin \theta+\frac{L_{\Delta}}{2}\left(i_{0} \sin 2 \theta+2 i_{0} \cos 2 \theta\right)\right] \\
& a_{31}=\frac{-n_{0}^{2}}{J}\left(K_{t} \sin \theta+\frac{L_{\Delta}}{2} i_{0} \sin 2 \theta\right), \\
& b_{33}=\frac{-n_{0}^{2}}{J}, \\
& b_{11}=\frac{-1}{2 L_{o}}\left(L_{\Sigma}-L_{\Delta} \cos 2 \theta\right), \\
& b_{12}=\frac{-L_{\Sigma}}{2 L_{o}} \sin 2 \theta, \\
& b_{21}=\frac{-L_{\Delta}}{2 L_{o}} \sin 2 \theta, \\
& b_{22}=\frac{1}{2 L_{o}}\left(L_{\Sigma}+L_{\Delta} \cos 2 \theta\right), \\
& a_{13}=\frac{K_{t}}{L_{0}} \sin \theta, \\
& a_{23}=\frac{K_{t}}{L_{0}} \sin \theta, \\
& a_{33}=\frac{-f}{J}
\end{aligned}
$$

and

$$
\begin{aligned}
& a_{14}=a_{24}=a_{34}=a_{41}=a_{42}=a_{44}=b_{13}= \\
& b_{23}=b_{31}=b_{32}=b_{41}=b_{42}=b_{43}=0
\end{aligned}
$$

The currents and the voltages are considered the inputs for the extended Kalman filter where as the position represents the output. In the Figure 4, a global representation of the vector control for the PMSM is given.

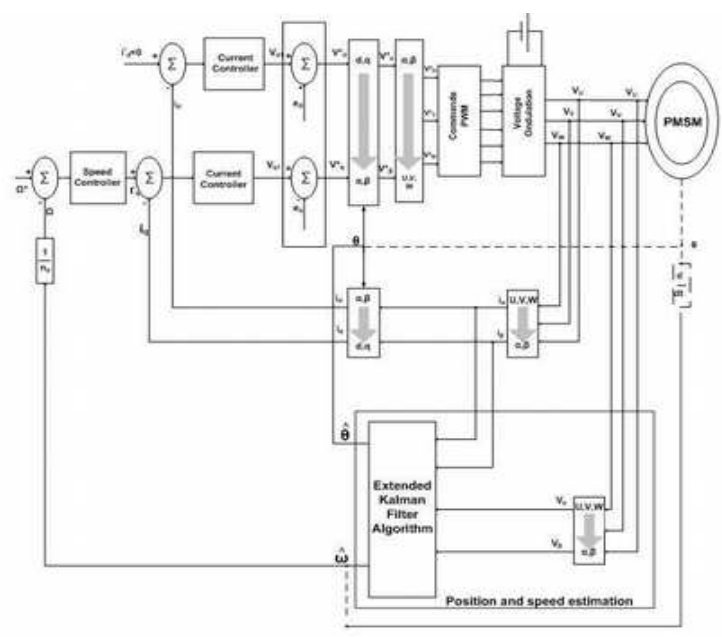

Figure 4. PMSM sensorless vector control

Different simulations, for several values of $\theta$, are developed in order to evaluate the EKF performances. 
The real (rp), the estimated (ep) positions and their error (e) for $\theta=0^{\circ}$ is given in Figure 5; the errors of the position for $\theta=60^{\circ}$ and $\theta=90^{\circ}$ are presented respectively in Figure 6 and Figure 7.

They show that the errors between real and estimated position decrease with the rotor position. The best result is obtained for $\theta=0^{\circ}$.
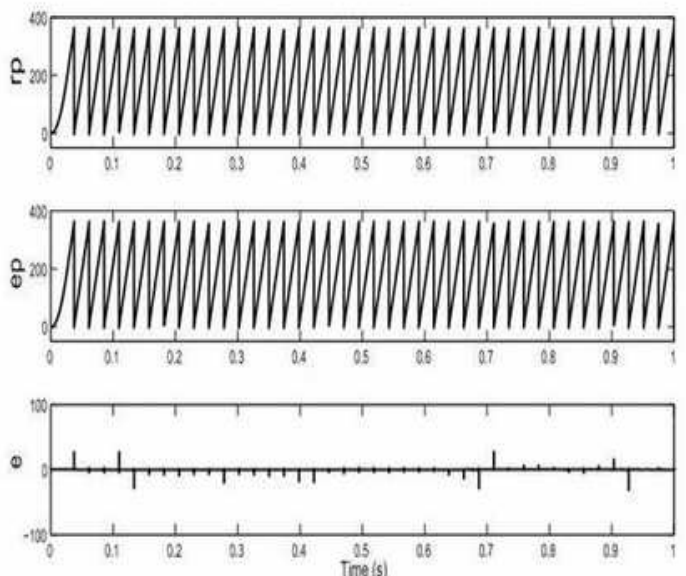

Figure 5. Evolutions of real $(r p)$ and estimated (ep) positions and $e=r p$-ep for a zero initial condition obtained with the FKE

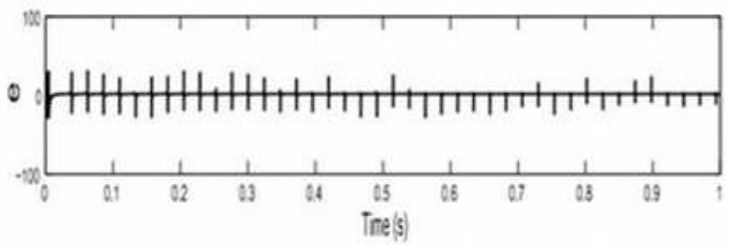

Figure 6. Position error (e) for the initial condition $\theta=60^{\circ}$

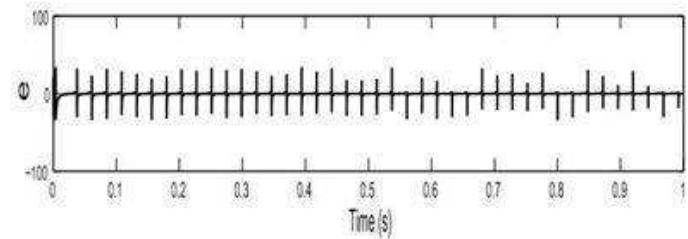

Figure 7. Position error (e) for the initial condition $\theta=90^{\circ}$

\section{Simulation Results}

The problem is to determine a feedback gain $K$ stabilizing the system for uncertain $R_{s}$, stator resistance $\pm 50 \% R_{s}$, and $J_{m}$, moment of inertia $\pm 50 \% J_{m}$, so that

$$
A=\left[\begin{array}{cccc}
-25.8 R_{s} & 60.6 & 0 & 0 \\
43.7 & -131.5 R_{s} & -13.8 & 0 \\
0 & 2.61 / J_{m} & -8.63410^{-4} / J_{m} & 0 \\
0 & 0 & 1 & 0
\end{array}\right]
$$

$B=10^{3}\left[\begin{array}{ccc}0.1828 & 0 & 0 \\ 0 & 0.1319 & 0 \\ 0 & 0 & -5 / J_{m} \\ 0 & 0 & 0\end{array}\right]$

To show the performances of the proposed ARE-GA formalism in terms of convergence, let run, several times, the implemented algorithm of optimization in order to obtain data on and $\mathrm{P}$ parameters.

Then, the best solutions of ARE-GA introduced in Figure 2, obtained by the use of Matlab 7.10, are

$$
\begin{aligned}
\varepsilon & =0.012 \\
P & =\left[\begin{array}{llll}
1.560 & 0.551 & 1.033 & 0.295 \\
0.551 & 1.318 & 0.122 & 1.093 \\
1.033 & 0.122 & 1.210 & 0.615 \\
0.295 & 1.093 & 0.615 & 3.263
\end{array}\right] .
\end{aligned}
$$

and the best solutions found by the use of ARE are:

$$
\begin{aligned}
\varepsilon & =0.032 \text { for } \varepsilon \text { in the interval }[0.001,1] \\
P & =\left[\begin{array}{llll}
1.230 & 0.412 & 0.962 & 0.512 \\
0.412 & 0.851 & 0.226 & 0.930 \\
0.962 & 0.226 & 1.103 & 0.416 \\
0.512 & 0.930 & 0.416 & 3.263
\end{array}\right] \\
K & =10^{3}\left[\begin{array}{llll}
0.4563 & 0.1318 & 0.4516 & 0.1976 \\
0.0543 & 0.1122 & 0.0298 & 0.1227 \\
3.3173 & 0.7793 & 3.8035 & 1.4345
\end{array}\right]
\end{aligned}
$$

For these gains, the stability is satisfied for the studied system.

By running 30 times the proposed ARE-GA algorithm, regardless of the initial population, we note that its convergence is always located in the same area of the search space. This means that the ARE-GA reaches each time the most interesting region of the search space. The results, obtained by simulation using the optimal settings of Figures 8, 9, 10 and 11, show the effectiveness of the proposed AREGA algorithm compared to the solution obtained by the Algebraic Riccati Equation (ARE).

\section{Conclusion}

In this paper, the proposed approach, based in the use of the ARE technique associated to GA, for solving a non-convex optimization problem, is formulated and tested, with success, and for the quadratic stabilizability study of the studied 


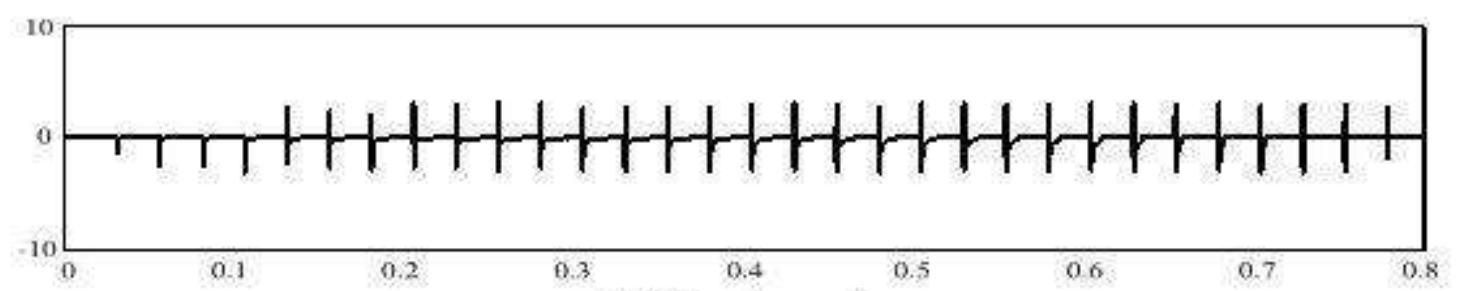

(a) Without correction

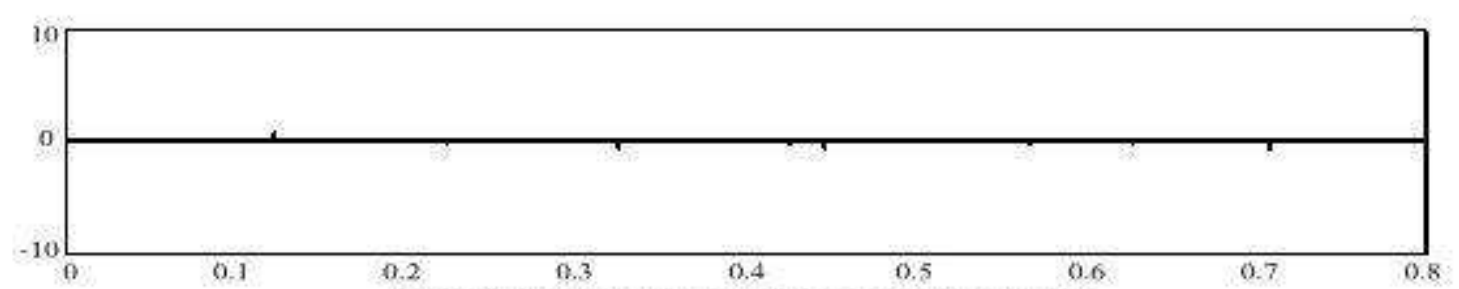

(b) with correction by convex optimization (ARE)

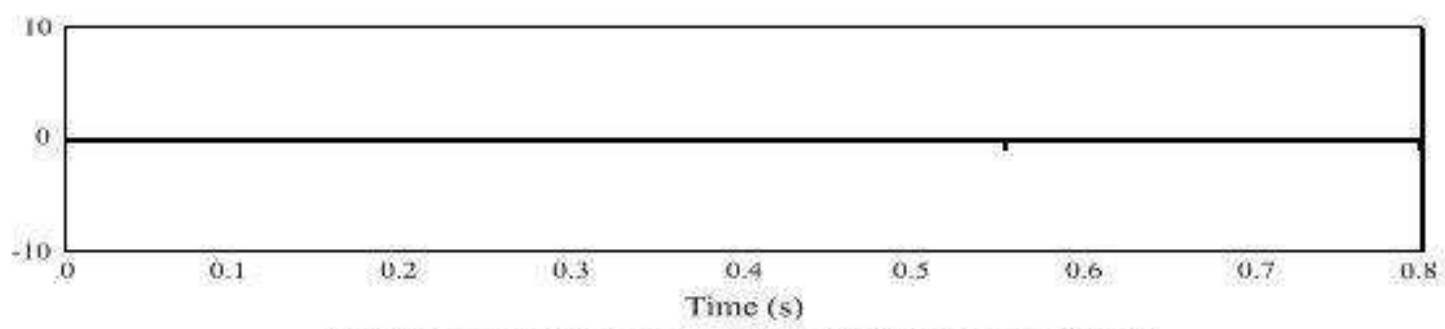

(c) with correction by non convex optimization (ARE-GA)

Figure 8. Position error $(e)$

for $-50 \% R_{s}$ and $+50 \% J_{m}$

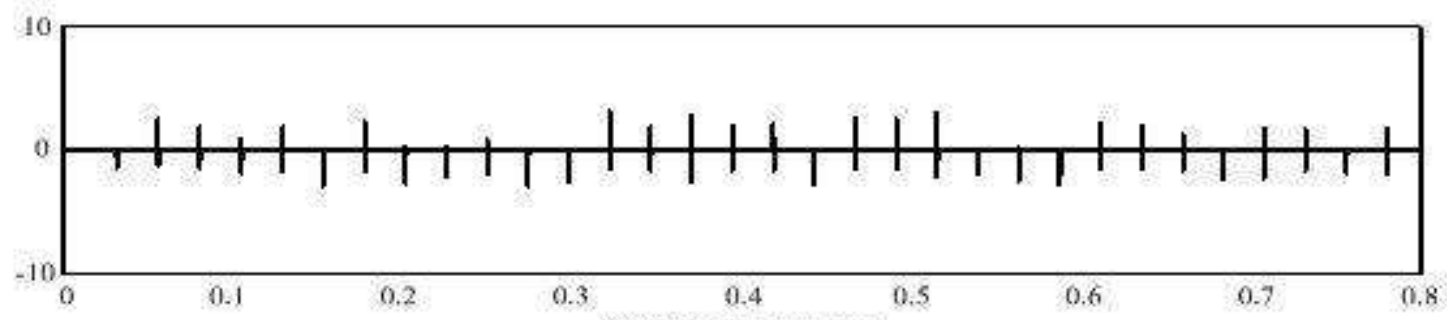

(a) Without correction
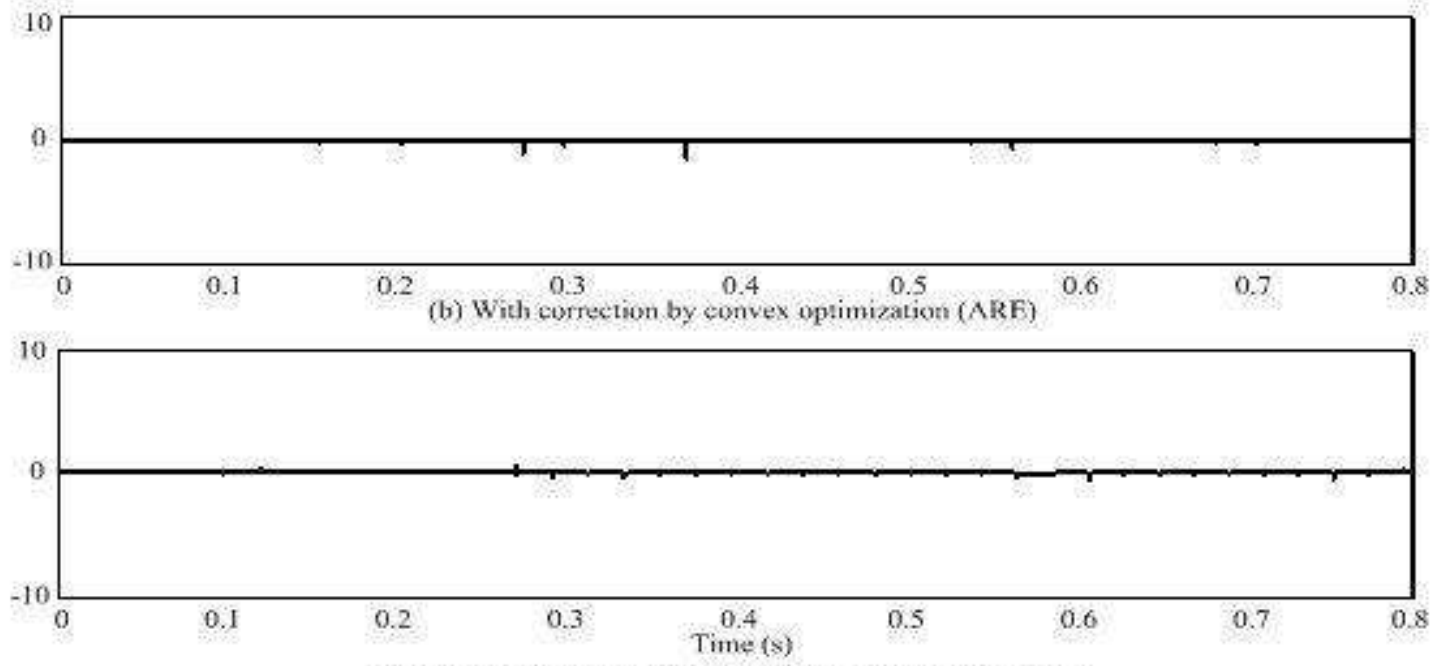

(c) With correction by nos convex optimization (ARE-GA)

Figure 9. Position error (e)

for $-50 \%$ Rs and $-50 \% \mathrm{Jm}$ 


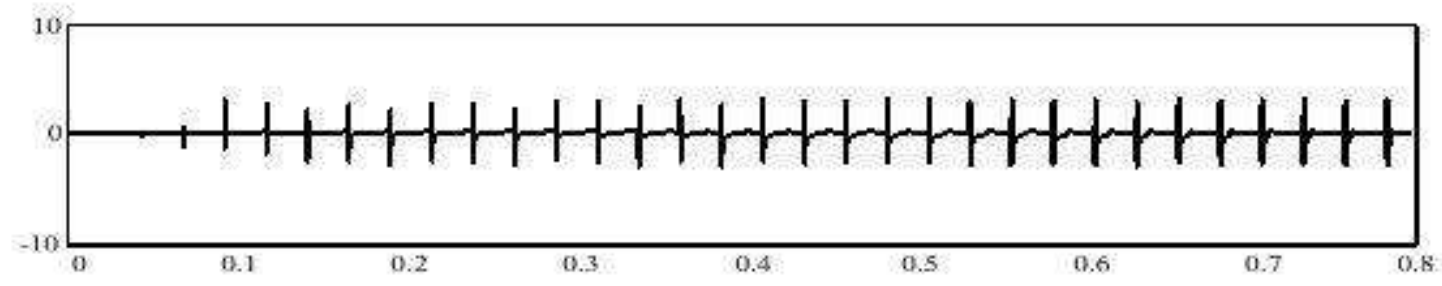

(a) Without correction

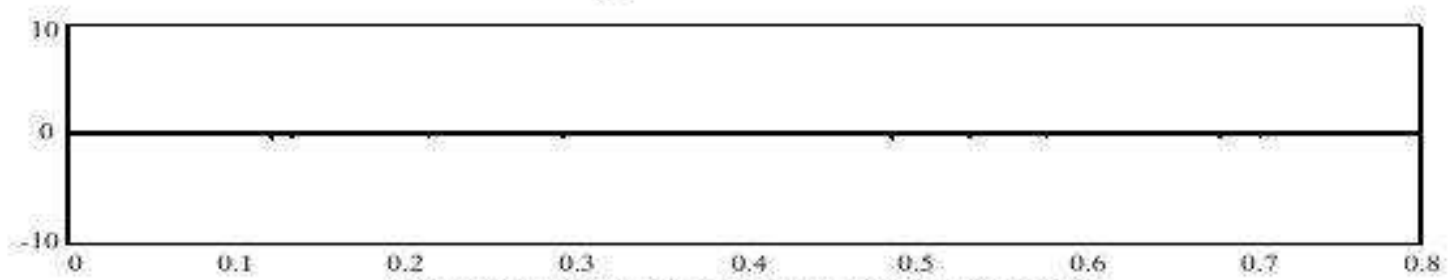

(b) with correction by convex optimization (ARE)

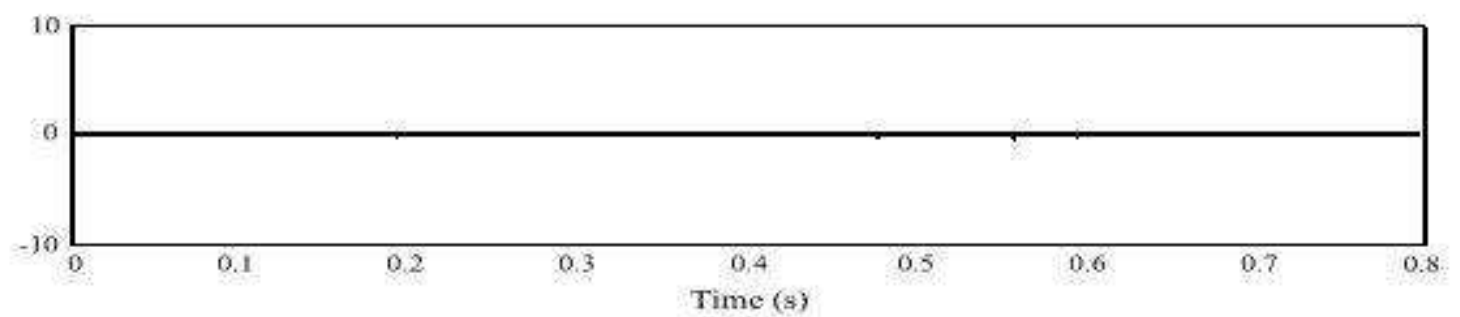

(c) with correction by non convex optimization (ARE-GA)

Figure 10. Position error $(e)$

for $+50 \% R_{s}$ and $+50 \% J_{m}$

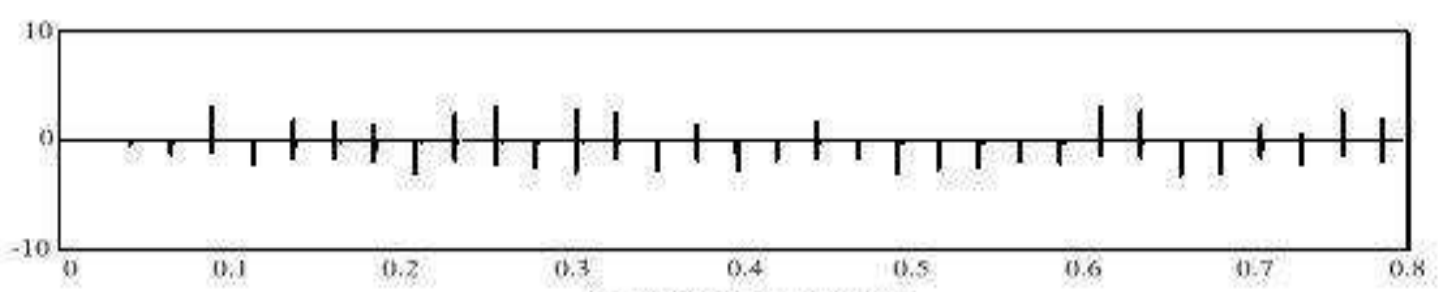

(a) Without correction

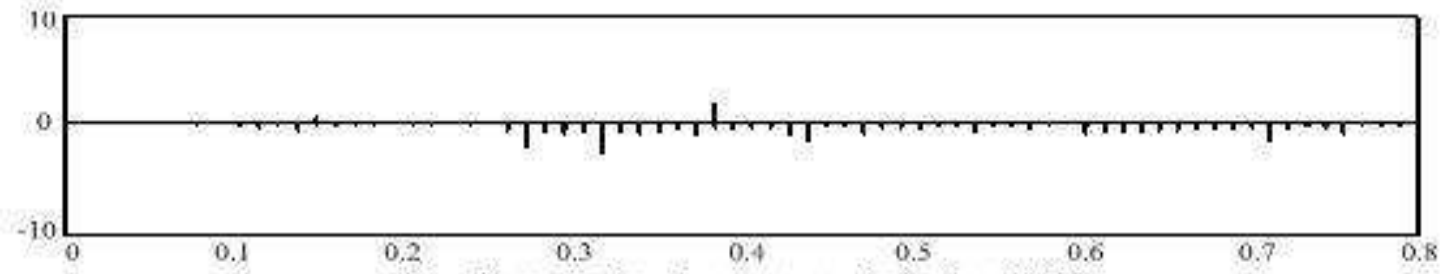

(b) with correction by convex optimization (ARLi)

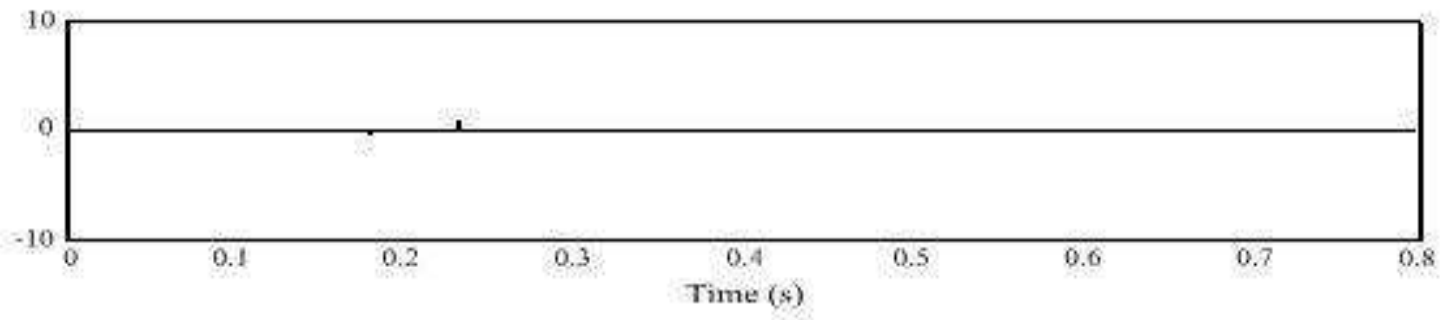

(c) with correction by non convex optimization (ARF.G.A)

Figure 11. Position error (e)

for $+50 \%$ Rs and $-50 \% \mathrm{Jm}$ 
PMSM. The EKF is used with success to control the position of PMSM. The obtained real estimated values of the position are comparable with real ones.

The comparison of these results with those obtained by the use of ARE technique shows that the best performances, in terms of speed convergence, quality solutions and simplicity implementation and robustness, are obtained when the proposed ARE-GA is applied.

\section{Acknowledgements}

The authors would like to thank the anonymous reviewers for their helpful comments and suggestions to improve the original manuscript.

\section{REFERENCES}

1. ROCKAFELLAR, R. T. Lagrange multipliers and optimality, SIAM Review vol. 35 , no. 2, 1993, pp. 183-238.

2. PÉREZ, E., ARINO, C. F. X. BLASCO, F. X., MARTINEZ, M. A., Maximal closed loop admissible set for linear systems with non-convex polyhedral constraints, Journal of Process Control, Science Direct, vol. 21, no. 4, April 2011, pp. 529-537.

3. BOUSSAK, M., Digital signal processor based sensorless speed control of a permanent magnet synchronous motor drive using extended Kalman filter, EPE Journal, vol. 11(Issue 3): 7-15, 2001.

4. CHBEB A., JEMLI M., BOUSSAK, M., GOSSA, M., Commande vectorielle d'un moteur synchrone à aimant permanent, Conférence JTEA, Hammamet, 2006.

5. BARUT, M., BOGOSYAN, S., GOKASAN, M., Speed-Sensorless Estimation for Induction Motors Using Extended Kalman Filters, IEEE Trans. on Ind. Electron., vol. 54, no 1, Feb. 2007.

6. BARMISH, B., Necessary and Sufficient Conditions for Quadratic Stabilization

7. of an Uncertain System, Journal Optimization Theory Appl., vol. 46, no. 7, 1985, pp. 399-408.

8. KHARGONEKAR, P., PETERSEN, I. R., ZHOU, K., Robust stabilization of uncertain linear systems: Quadratic stability and $\boldsymbol{H}_{\infty}$ control theory, IEEE
Trans. on Autom. Contr., vol. 35 no. 3, 1990, pp. 356-361.

9. FARAG, A., WERNER, H., A Riccati genetic algorithms approach to fixedstructure controller synthesis, American Control Conference, Boston, Massachusetts, June, 2004.

10. ROJAS, A. J., Closed-form solution for a class of continuous-time algebraic Riccati equations, 48th IEEE Conference on Decision and Control, Shanghai, Dec. 2009.

11. CARRIERE, S., CAUX, S., FADE, M., Synthèse LQ pour le contrôle en vitesse d'un actionneur synchrone autopiloté accouplé directement à une charge mécanique incertaine, Revue e-STA, vol. 6 , no. $1,2009$.

12. CHENG, D., MARTIN, C., XIANG, J., An Algorithm for Common Quadratic Lyapunov Function, The 3rd World Congress on Intelligent Control and Automation, Hefei, P. R, 2000.

13. SOMYOT, K., MANUKID, P., GeneticAlgorithm-Based Fixed-Structure Robust $\mathrm{H}_{\infty}$ Loop Shaping Control of a Pneumatic Servosystem. Journal of Robotics and Mechatronics, vol. 16, no. 4, 2004, pp. 362-373.

14. BORNE, P., BENREJEB, M., Des algorithmes d'optimisation. La nature, source d'inspiration pour l'ingénieur. L'essor des "métaheuristiques pour l'optimisation difficile" basées sur le comportement de la nature. L'ingénieur, (260), 2010, pp. 12-15.

15. CHEN, B-S., CHENG, Y-M., A Structured-Specified $\mathrm{H}_{\infty}$ Optimal Control Design for Practical Applications: A Genetic Approach, IEEE Transactions on Control Systems Technology, vol. 6, no. 6, 1998, pp. 707-718.

16. GOLDBERG, D. E., Algorithmes génétiques : exploration, optimisation et apprentissage automatique. AddisonWisley, France, 1994.

17. HOLLAND, J. H., Adaptation in Natural and Artificial Systems, University of Michigan Press, Ann. Arbor, 1975. 
18. JUNGERS, M., Jeux différentiels LQ de Stackelberg avec une pondération temporelle commune, Revue e-STA, vol. 3 , no. 4, 2006.

19. PETERSEN, I. R., A Stabilization algorithm for a class of uncertain linear systems, Systems and Control Letters, vol. 8,1987 , pp. 351-357.

20. FAKHARIAN, A., HAMIDI BEHESHTI, M.T., NAJAFI, M., A New Algorithm for Solving Riccati Equation Using Adomian Decomposition Method, Proceedings of the 9th WSEAS International Conference on Applied Mathematics, Istanbul, Turkey, May 27-29, 2006, pp.74-77.

21. TSEVEENDORJ, I., FORTIN, D., Global Optimization and Multik-napsack: a percolation algorithm, European Journal of Operational Research, vol. 154, Issue 1, April 2004, pp. 46-56.

22. ZHOU, K., KHARGONEKAR, P., Robust stabilization of linear system norm bounded time-varying uncertainty, Systems and Control Letters, vol. 10, 1988, pp. 17-20.

23. GARCIA, G., BERNUSSOU, J., $\mathrm{H}_{2}$ guaranteed cost design by output feedback, SIAM J. of Control and Optimization, 1995.

24. COLMINARES, W., Sur la robustesse des systèmes linéaires incertains: Approche quadratique, retour de sortie, Thèse Docteur-Ingénieur, Université Paul Sabatier de Toulouse, 1996.

25. SUBOTIC, M., TUBA, M., Parallelized Multiple Swarm Artificial Bee Colony Algorithm (MS-ABC) for Global Optimization, Studies in Informatics and Control, ISSN 1220-1766, vol. 23 (1), 2014, pp. 117-126.

26. CARLOTA, C., CHA, S., On existence of solutions for nonconvex optimal control

27. problems, Mathematical Methods in Science and Mechanics, 2014, pp. 70-73.

28. LUIS DIAS PERES, P., Sur la robustesse des systèmes linéaires: Approche par programmation linéaire, $\mathrm{PhD}$, Université de Paul Sabatier de Toulouse, 1989.
29. CHRYSSOVERGHID, I., DiscretizationOptimization Methods for Optimal Control Problems, Proceedings of the 5th WSEAS Int. Conf. on SIMULATION, MODELING AND OPTIMIZATION, Corfu, Greece, August, 17-19, 2005, pp. 399-406.

30. MAO, W. J., CHU, J., Quadratic Stability and Stabilization of Dynamic Interval Systems, IEEE Transactions on Autom. Contr., vol. 48, no. 6, June 2003.

31. DCHICH, K., ZAAFOURI, A., CHBEB, A., JEMLI, M., Position sensorless robust control of PMSM using the extended Kalman filter algorithm, International Review on Modelling and Simulations (I.RE.MO.S.), vol. 6, no 2, April 2013, pp. 380-386.

32. NICOARĂ, E. S., FILIP, F. G., PARASCHIV, N., Simulation-based Optimization using Genetic Algorithms for Multi-objective Flexible JSSP, Studies in Informatics and Control, ISSN 1220-1766, vol. 20 (4), 2011, pp. 333-344.

33. HU, T., TEEL, A. R., ZACCARIAN, L., Non-quadratic Lyapunov functions for performance analysis of saturated systems, EEE Conference on Decision and Control, The European Control Conference 2005, Séville, December 2005.

34. TSEVEENDORJ, I., Conditions d'optimalité en optimisation globale: contributions à l'optimisation combinatoire, $\mathrm{PhD}$, Université de Versailles, St. Quentin en Yvelines, 2007.

35. BARMISH, B., Stabilization of Uncertain Systems via Linear Control, IEEE Trans. on Autom. Control, vol. AC 28, no. 8, August 1983, pp. 848-850. 